\title{
Migraine aura-like headache in occipital lobe brain tumors
}

\author{
Domenico Chirchiglia, Pasquale Chirchiglia, Rosa Marotta
}

Department of Neurosurgery, University of Catanzaro, Italy

\begin{abstract}
Background. Headache is a common symptom in brain tumors. In agreement with the International Headache Society, tumor headache belongs to the headache attributed to neoplasia. It has nonspecific characteristics regarding the type of pain that is diffused or localized in correspondence with the site of the tumor. In this study, we describe two cases of migraine-like headache in occipital lobe tumors, hypothesizing possible mechanisms involving cortical spreading depression.

Methods. The study concerns a series of 38 brain tumors, with headache as an onset symptom. It had the characteristics of a traction headache, typical of brain tumors. Headache occurred with typical migraine symptoms in two occipital lobe tumors. This is a singular occurrence, observing the literature data. The patients underwent surgical treatment, with the disappearance of the headache.

Results. This study showed the correlation between migraine aura-like headache and occipital lobe brain tumor. Migraine headache was the onset symptom in the patients affected by occipital lobe brain tumors. They underwent surgical treatment with disappearance of headache.

Conclusions. In the cases described in this work, a correlation was found between the occipital tumor and migraine-like headache. It is a singular case, confirming the involvement of the occipital cortex in migraine with visual aura-related headache, through the start of spreading depression.
\end{abstract}

Keywords: migraine, brain tumors, occipital lobe, headache

\section{INTRODUCTION}

Headache is one of the most frequent symptoms in brain tumors, according to some studies the onset symptom [1]. It is known as a late-onset headache must make suspect of the presence of a mass within the brain. Headache in brain tumors has nonspecific features, presenting spread to the entire head, or localized to the area affected by the tumor. The pain can be described as a weight on the surface of the skull, in particular at its center, or constrictive, described as a feeling of oppression on the temporal regions [2]. International Headache Society (IHS) codified and inserted this type of headache among those secondary to brain tumors. The duration is variable, influenced by the clinical status of the patient, as well as the frequency, going from one episode per month to one per week $[2,3]$. We have conducted a study on headache, as a symptom of a series of brain tumors, before surgery, with the aim of describing the features, such as type, duration, frequency, correlation with the type of tumor.

\section{MATERIAL AND METHODS}

We analyzed retrospectively 38 cases of brain tumors, 20 males and 18 females, aged between 40 and 71 years, average 51.5 . The tumors were represented by 15 low-grade astrocytomas, 3 glioblastomas, 10 meningiomas, 10 metastases. About location parietal tunors were 12, frontal tumors 16 , temporal tumors 8 , occipital tumors 2 . All patients had headaches as tumor onset. Headache had the 
following characteristics: pain localized throughout the head or in some regions, described as a weight or constricting, frequency 1-2 episodes per month, duration several hours, not always responsive to the drugs. Tumor headache is the so-called traction headache due to stretching of the cerebral and dural arterial vessels. In two patients, a female affected by occipital meningioma and a male affected by low-grade occipital astrocytoma, the headache had the features of migraine with aura. The pain was pulsating, unilateral, associated with nausea, vomiting, phono-photophobia, duration from hours to days, frequency one attack per month, while the visual aura was represented by phosphenes and teicopsias. Neither of them had ever suffered from migraine which had appeared a few months before the discovery of the tumor.

\section{RESULTS}

After completing the diagnostic tests, overall neuroimaging examinations (brain CT-MRI), we decided to undertake the surgical option. So we acted in the following way: of the 38 patients, 30 were subjected to surgery, with disappearance of the headache, including the two patients with migraine-like headache.

\section{DISCUSSION}

Headache in brain tumors is a frequent symptom. IHS has inserted this type of headache between headaches attributed to non-vascular intracranial disorders (7.4. Headache attributed to intracranial neoplasia). This headache is linked in close relation to the appearance of the tumor, can be accompanied by vomiting, worsens in the morning. The prevalence is estimated to be between 32 and $71 \%$ and it favors the young age $[3,4]$. The pain is localized in every part of the head and described as something that weights on the head or tightens the head $[5,6]$. It's certainly not a migraine pain. In our case series we found two cases of migraine aura-like headache that preceded the discovery of the tumor. Migraine has been associated with a brain tumor if pre-existing [7]. Some cases of migraine-like headache in brain tumors have been described in literature. Forsyth in a study of 111 cases of brain tumors found headache in $48 \%, 77 \%$ simi- lar to the tension-type headache, only $9 \%$ migraine aura-like headache [8]. Instead Kurth, studying 13 cases of women with brain tumors, found as an anticipatory sign migraine headache in 7 of them [9]. Galletti et al., in an observational study of the clinical and angiographic characteristics of a cohort of 40 consecutive patients with arteriovenous malformations (AVMs), found migraine-like headache as the first clinical manifestation in $22.5 \%$ of patients. The location of the malformation was significantly associated with migraine-like presentation and the occipital lobe was the predominant site. Headache associated with arteriovenous malformations in the occipital lobe, although secondary in nature, could have clinical features similar to migraine. The hypothesis was that an occipital location may be linked with spreading depression, a pathogenic mechanism of migraine [10]. Shams et al. presented 9 patients and a further 31 cases from the literature who experienced visual aura fulfilling the diagnostic criteria for migraines but caused by focal occipital pathology concluding that any disease process that is able to create a state of neuronal hyperexcitability can therefore increase an individual's susceptibility to the development of cortical spreading depression, the electrophysiological correlate of the visual aura [11].

Another case of occipital lobe tumor presenting as migraine with typical aura was reported in a woman by Verma A. [4]. In our case studies about the tumor's location, the two patients suffering from occipital lobe brain tumor, had migraine aura-like headache. We know that a tumor of the occipital lobe causes vision disturbances, similar to the signs of the migraine aura. This could be an explanation for the onset of a migraine aura-like headache in an occipital lobe brain tumor. It is known that aura in migraine is related to the involvement of the occipital cortex, through the wellknown phenomenon of the cortical spreading depression, causing visual disturbances, followed by activation of the trigeminal-vascular system, responsible for migraine pain. Cortical spreading depression (CSD) or spreading depolarization is a wave of electrophysiological hyperactivity followed by a wave of inhibition. Spreading depolarization is a phenomenon characterized by the appearance of depolarization waves of the neurons and neuroglia, propagating from occipital areas 
across the cortex at a velocity of $2-5 \mathrm{~mm} / \mathrm{min}$. CSD can be induced by hypoxic conditions and facilitates neuronal death. It has long been recognized the role of CSD in the genesis of migraine aura. This correlation strengthens the hypothesis of the mechanisms underlying the onset of migraine with visual aura, in which there is the involvement of the occipital lobe.

\section{CONCLUSIONS}

Migraine aura-like headache as a sign of occipital lobe brain tumor is a singular occurrence, which offers several hypotheses about this relationship. Usually, brain tumor headaches are described as headaches with features more similar to tension-type headaches than to migraines. Therefore, it is strongly suggestive that the mechanism of spreading depression can initiate the symptoms of visual aura even in occipital brain tumors.

Conflict of interest: none declared Financial support: none declared

\section{REFERENCES}

1. Taylor PL. Mechanism of brain tumor headache. Headache 2014. 54(4):772-5.

2. The International Classifications of Headache Disorders, 3rd edition, 2018

3. Shimizu Y, Yamane K, Tsutsumi $Y$ et al. A case of migraine with aura associated with meningioma. Rinsho Shiukeigaku 1993. 33 (4):396-9.

4. Verma A, Rosenfeld V, Forteza A et al. Occipital lobe tumor presenting as migraine with typical aura. Headache, 1996 jan, 36(1):49-52

5. Evans RW, Timm JS, Baskin DS. A Left Frontal Secretory Meningioma Can Mimic Transformed Migraine With and Without Aura. Headache 2015. 55(6):849-52.

6. Sakakibara Y, Taguchi $Y$, Uchida K. A case of cavernous angioma presenting as migrainous attack. No Shinkei Geka 2010. 38(3):287-91.

7. Purdy RRA, Kirby S. Headaches and brain tumors. Neurol Clin 2004. 22(1):39-53.

8. Forsyth PA, Posner JB. Headaches in patients with brain tumors: A study of 111 patients. Neurology 1993. 43(9):1678-83.

9. Kurth T, Buring JE, Rist PM. Headache, migraine and risk of brain tumors in women: prospective cohort study. J Headache Pain 2015:16:51

10. Galletti F, Sarchielli P, Hamam M, Costa C, Cupini LM, Cardaioli G, Belcastro V, Eusebi P, Lunardi P, Calabresi P. Occipital arteriovenous malformations and migraine. Cephalalgia 2011. 31(12):1320-4.

11. Shams PN, Plant GT. Migraine-like visuali aura due to focal cerebral lesions: Case series and review. Surv Ophthalmol. 2011. 56(2):13561. 